\title{
Pharmacokinetics of Aniracetam and Its Metabolites in Rat Brain
}

\author{
Taro Ogiso, ${ }^{*, a}$ Kaori Uchiyama, ${ }^{a}$ Hiroko Suzuki, ${ }^{a}$ Mika Yoshimoto, ${ }^{a}$ Tadatoshi Tanino, ${ }^{a}$ \\ Masahiro IwAKI, ${ }^{a}$ and Satoshi $\mathrm{UNO}^{b}$ \\ Faculty of Pharmaceutical Sciences, Kinki University," 3-4-1 Kowakae, Higashi-Osaka, Osaka 577-8502, Japan and \\ Azwell Co., ${ }^{b}$ 2-24-3, Sho, Ibaraki, Osaka 567-0806, Japan. Received November 4, 1999; accepted December 27, 1999
}

\begin{abstract}
The pharmacokinetics of aniracetam (AP) and its main metabolites, 4-p-anisamidobutyric acid (ABA), 2pyrrolidinone (PD) and $p$-anisic acid (AA), in 3 brain regions (cerebral cortex, hippocampus and thalamus) was investigated after single intravenous (i.v.) and oral administrations of AP to rats. AP, AA and PD were rapidly distributed into the 3 brain regions after i.v. administration of AP, but the amounts of AP were low. The concentrations of $\mathrm{AP}$ and $\mathrm{AA}$ in brain regions rapidly declined, whereas $\mathrm{PD}$ levels were higher and more sustained than those of AP and AA. ABA levels in the regions were below the detection limit. There were no significant differences in the distribution of these compounds in the 3 brain regions. The $A U C_{\text {brain }} / A U C_{\text {plasma }}$ ratio of PD was 53$55 \%$, in contrast to the low ratio of AP $(2.4-3.2 \%)$ and $A A(3.9-4.2 \%)$. On oral administration of AP, the $A U C_{\text {brain }} / A U C_{\text {plasma }}$ ratio of $\mathrm{PD}$ was also higher than that of $\mathrm{AA}$. When the transport of $\mathrm{PD}$ was tested using the in situ brain perfusion technique, it was clarified that PD was not transported across the blood-brain barrier (BBB) by a neutral amino acid carrier system. The high brain levels of PD and the low levels of AP suggest that the clinical efficacy of dosed AP may partly result from PD penetrating into the brain.
\end{abstract}

Key words aniracetam; metabolite; brain pharmacokinetics; brain concentration; brain uptake

Aniracetam (1- $p$-anisoyl-2-pyrrolidinone, AP), a member of the group of cyclic $\gamma$-aminobutyric acid (GABA)-derivatives, is a new drug for improving cerebral insufficiency. AP is widely used as a cognitive performance enhancer for cerebral embolism in Japan. In a previous paper, we reported pharmacokinetic analysis of the plasma levels of AP and its metabolites using a pharmacokinetic model with 7 compartments, and it was found that plasma concentrations of 2 pyrrolidinone (PD), a metabolite of AP, were much higher and more sustained than those of AP. ${ }^{1)}$ Of particular interest is the observation that PD is a pharmacologically active (PD shortened the choice reaction time at doses of $10-100$ $\mathrm{mg} / \mathrm{kg}$, which are equivalent to the doses of AP). ${ }^{2,3)}$ Therefore, it is suggested that the clinical efficacy of administered AP may partly result from PD formed from the parent drug. Although the distribution of ${ }^{14} \mathrm{C}$-AP into brain after oral administration has been reported in rats, ${ }^{4,5}$ the distribution of parent drug and its metabolites in the brain and their disposition from the regions have not yet been fully elucidated.

In this paper, the pharmacokinetics of $\mathrm{AP}$ and its main metabolites, 4- $p$-anisamidobutyric acid (ABA), PD and $p$ anisic acid (AA) in the brain were investigated after single intravenous (i.v.) and oral administrations of AP to rats. The second objective of this work was to characterize the putative transport carrier for PD which distributed it to the brain at higher levels after i.v. administration of AP.

\section{MATERIALS AND METHODS}

Materials AP and ABA were generous gifts from JapanRoche Co. (Tokyo, Japan). AA and PD were purchased from Aldrich Chemical Co. (Milwaukee, WI) and Sigma Chemical Co. (St. Louis, MO, U.S.A.), respectively. Ethyl $p$-hydroxybenzoate and $N$-methyl-2-pyrrolidone, internal standards for the HPLC, were obtained from Nacalai Tesque Co. (Kyoto, Japan). Dextran sulfate (average M.W. 500000) was obtained from Sigma Chemical Co. L-Alanine, L-proline, L-leucine, Lphenylalanine and 3,4-dihydroxyphenylalanine (L-DOPA)

* To whom correspondence should be addressed. were obtained from Wako Pure Chemical Industries Co. (Osaka, Japan). All other chemicals and solvents used were of reagent or HPLC quality.

Animals Male Wistar rats, weighing 220-250g, were used throughout this experiment. They were maintained in a controlled temperature $\left(24 \pm 1^{\circ} \mathrm{C}\right)$ and light $(12 \mathrm{~h} \mathrm{light} / 24 \mathrm{~h})$ environment. A group of $3-5$ rats was randomly selected from the population. On the day before the experiment the rat jugular vein was cannulated with a silicon tubing.

I.v. and Oral Administrations AP $(30 \mathrm{mg} / \mathrm{kg})$ was administered to one group of rats intravenously through the cannula as a solution $(0.15 \mathrm{ml} / 100 \mathrm{~g})$ in a mixture of propylene glycol-ethanol-water $(5: 2: 3, \mathrm{v} / \mathrm{v})$. Another group of rats received AP $(50 \mathrm{mg} / \mathrm{kg})$ orally as an aqueous suspension $(0.5 \mathrm{ml} / 100 \mathrm{~g})$ in $1.5 \%$ carboxy methyl cellulose (CMC). Rats were sacrificed by decapitation at 15, $30 \mathrm{~min}, 1$ and $2 \mathrm{~h}$ after i.v. dosing, and 15, 30, $45 \mathrm{~min}, 1,2$ and $4 \mathrm{~h}$ after oral dosing. The brain was rapidly removed and dissected into 3 regions, cerebral cortex, hippocampus and thalamus. Each brain region was weighed (cerebral cortex $0.33-0.39 \mathrm{~g}$, hippocampus $0.031-0.054 \mathrm{~g}$ and thalamus $0.12-0.16 \mathrm{~g}$ ) and homogenized for $2 \mathrm{~min}$ (glass homogenizer) after adding 10 volumes of $0.1 \mathrm{~m}$ sodium dihydrogen phosphate under chilling.

In Situ Rat Brain Perfusion PD transport at the bloodbrain barrier $(\mathrm{BBB})$ was investigated using the in situ rat brain perfusion technique developed by Takasato et al. ${ }^{6)}$ Briefly, the rat was anesthetized with pentobarbital (40 $\mathrm{mg} / \mathrm{kg}$ ) and the right common carotid and right external carotid arteries were isolated. The external carotid artery was catheterized for retrograde perfusion with a $15-\mathrm{cm}$ segment of PE-50 tubing without interruption of blood flow to the brain. A syringe containing perfusate at $37^{\circ} \mathrm{C}$ was connected to the cannula. The common carotid artery was ligated to direct perfusate flow through the internal carotid artery and onto the brain capillaries. The rat brain was perfused for 1 $\mathrm{min}$ at a flow rate of $4.98 \mathrm{ml} / \mathrm{min}$ with either saline containing PD $(2.0 \mathrm{mg} / 5 \mathrm{ml})$ or PD and $0.1 \mathrm{M}$ amino acid but $0.015 \mathrm{M}$ 
Cerebral cortex Hippocampus Thalamus
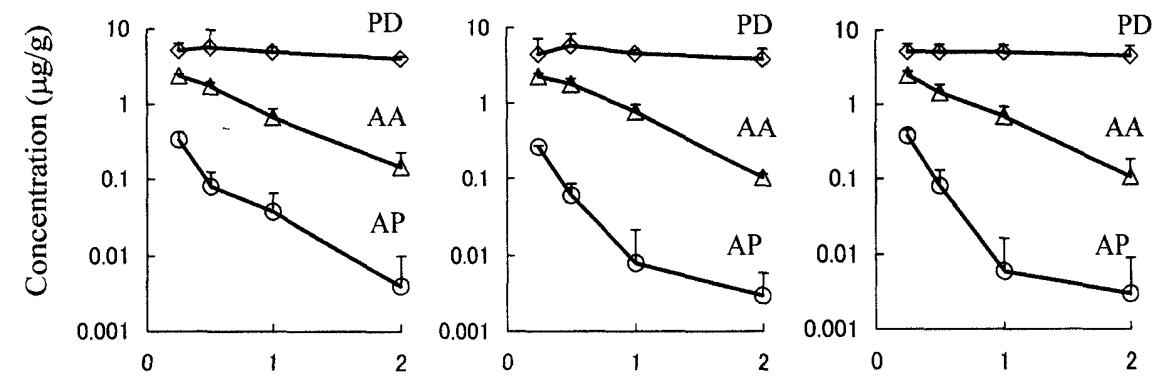

Time (h)

Fig. 1. Brain Concentration-Time Curves for AP and Its Metabolites after I.v. Administraton of AP

The dose of AP was $30 \mathrm{mg} / \mathrm{kg}$. Points and vertical bars represent the mean \pm S.D. of 3 rats.

L-DOPA, using a Harvard microprocessor syringe pump 22. The perfusion time ( $1 \mathrm{~min}$ ) was decided based on the result presented by Takasato et al. ${ }^{6)}$ At the end of perfusion, the rat was sacrificed by decapitation. The brain was quickly freed from cranium, and the perfused half (right) brain hemisphere was placed on an ice-chilled glass plate. The cerebral cortex was homogenized in 10 volumes of $0.1 \mathrm{M}$ sodium dihydrogen phosphate under chilling.

Determination of AP, ABA, AA and PD AP, ABA and $\mathrm{AA}$ in the brain were simultaneously determined by the HPLC method. A $500 \mu 1$ aliquot of the homogenate was mixed with $100 \mu 1$ of ethyl $p$-hydroxybenzoate solution (2 $\mu \mathrm{g} / \mathrm{ml}$ chloroform). After adding $2.0 \mathrm{ml}$ of $0.1 \mathrm{~mm}$ dextran sulfate (average M.W. 500000) to remove lipids and $5 \mathrm{ml}$ of chloroform, the resulting mixture was shaken for $15 \mathrm{~min}$ and centrifuged at $3000 \mathrm{rpm}$. The organic phase was evaporated to dryness under reduced pressure and the residue was dissolved in $100 \mu \mathrm{l}$ of the elution solvent. After filtration through a membrane filter $(0.45 \mu \mathrm{m}$, Ekicrodisc $3 \mathrm{CR}$, Gelman Sciences Japan, Tokyo, Japan), the filtrate was injected onto a reversed-phase Inertsil octadecyl silica (ODS) column $(4.6 \times 150 \mathrm{~mm}$, GL Sciences Inc., Tokyo, Japan) using a Shimadzu liquid chromatography (model LC-6A) equipped with a UV spectrophotometer (model SPD-6AV). For the determination of $\mathrm{PD}$, a $400 \mu \mathrm{l}$ aliquot of the homogenate was mixed with $10 \mu \mathrm{l}$ of chloroform containing $N$-methyl-2-pyrrolidone ( $20 \mu \mathrm{g} / \mathrm{ml}), 200 \mu \mathrm{l}$ of $0.4 \mathrm{M}$ perchloric acid containing $0.05 \%$ sodium EDTA, $2.0 \mathrm{ml}$ of $0.1 \mathrm{~mm}$ dextran sulfate and $5 \mathrm{ml}$ of chloroform. The resulting mixture was shaken and centrifuged. The evaporation and dissolution were carried out by the same method as mentioned above. The filtrate was injected onto a reversed-phase Cosmosil $5 \mathrm{C} 18$ column (4.6X $150 \mathrm{~mm}$, Nacalai Tesque Co.). The mobile phase for determination of AP, ABA and AA was acetonitrile- $\mathrm{H}_{2} \mathrm{O}$-acetic acid $(25: 75: 0.5, \mathrm{v} / \mathrm{v})$ and for PD was methanol- $\mathrm{H}_{2} \mathrm{O}(5: 95$, $\mathrm{V} / \mathrm{v})$, pumped at a flow rate of $1.0 \mathrm{ml} / \mathrm{min}$. Detection was 254 $\mathrm{nm}$ for AP, ABA and AA and $205 \mathrm{~nm}$ for PD. The calibration curves of these compounds were determined by the same method using brain homogenates and the identical reagents. The detection limit for all compounds was $10 \mathrm{ng} / \mathrm{g}$. Intra- and interday variabilities were $<10 \%$.

Data Analysis The area under the concentration-time curve $(A U C)$ up to the last sampling point was calculated by the trapezoidal method. The area under the first moment curve $(A U M C)$ and the mean residence time $(M R T)$ up to the last sampling point were calculated by means of moment analysis. ${ }^{7)}$ The apparent bioavailability $(F)$ of parent drug in brain were calculated as

$$
F=\left[A U C_{\text {brain, } 0-4, \text { p.o. }} \times \operatorname{dose}_{\text {i... }} / A U C_{\text {plasma, } 0-4, \text { i.v. }} \times \operatorname{dose}_{p . o}\right] \times 100
$$

where $A U C_{\text {brain, } 0-4, \text { p.o. }}$ and $A U C_{\text {plasma, } 0-4 \text {, i.v. }}$ are the $A U C_{0-4}$ of AP in brain after oral dosing and in plasma after i.v. dosing of parent drug, respectively. The elimination rate constant $(k)$ was calculated from the slope of the plot.

The means of all data are presented with their standard deviation (mean \pm S.D.). Statistical analysis was performed using a unpaired Student's $t$-test, and the significant level adopted was $p<0.05$.

\section{RESULTS AND DISCUSSION}

I.v. Administration The time courses of AP, PD and AA in 3 brain regions (cerebral cortex, hippocampus and thalamus) after i.v. administration of AP at a dose of $30 \mathrm{mg} / \mathrm{kg}$ are shown in Fig. 1. The pharmacokinetic parameters are summarized in Table 1. There were no significant differences in the distribution of these compounds among the three brain regions. The concentrations of $\mathrm{PD}$ in brain were much higher and more sustained than those of AP and AA. As a consequence, MRT for PD was greater than that for AP and AA. AP and AA rapidly declined from these regions, with high $k_{\mathrm{e}}$ values. ABA levels in the brain regions were under the detection limit of the assay. Judging from the plasma concentrations of these compound after i.v. dosing of AP reported previously, ${ }^{1)}$ it is considered that their distribution into the brain was approximately in proportion to the plasma levels, with the exception of PD, and that they rapidly distributed into the regions, although the concentrations in the brain were much lower than those in the plasma. The $A U C_{\text {brain }}$ was compared with the $A U C_{\text {plasma }}$ obtained previously. ${ }^{1)}$ The $A U C_{\text {brain }} / A U C_{\text {plasma }}$ ratios of $\mathrm{AP}, \mathrm{PD}$ and $\mathrm{AA}$ were $2.4-3.4,53.0-54.8$ and $3.9-4.2 \%$, respectively (Table 1 ). High values of PD, which were over 10 times the values of AP and AA, were noticed. The distribution of PD into the brain exceeded that of more lipophilic compounds, such as AA (soluble in 2500 parts water, freely soluble in chloroform and ether ${ }^{8)}$ ). This fact suggests that PD might be mainly, but not exclusively, transported across the rat $\mathrm{BBB}$ to brain by a carrier-mediated 
Table 1. Pharmacokinetic Parameters of AP and Its Metabolites in Brain after I.v. Administration of AP ${ }^{a)}$

\begin{tabular}{|c|c|c|c|c|c|c|}
\hline & \multicolumn{3}{|c|}{ AP } & \multicolumn{3}{|c|}{$\mathrm{AA}$} \\
\hline & Cerebral cortex & Hippocampus & Thalamus & Cerebral cortex & Hippocampus & Thalamus \\
\hline \multirow{6}{*}{$\begin{array}{l}A U C_{0-2}(\mu \mathrm{g} \cdot \mathrm{h} / \mathrm{g}) \\
M R T(\mathrm{~h}) \\
A U C_{\text {brain }} / A U C_{\text {plasma }}(\%)^{b)} \\
k_{e}\left(\mathrm{~h}^{-1}\right)\end{array}$} & $0.145 \pm 0.018$ & $0.102 \pm 0.021$ & $0.134 \pm 0.017$ & $1.91 \pm 0.25$ & $1.92 \pm 0.19$ & $1.79 \pm 0.23$ \\
\hline & $0.47 \pm 0.06$ & $0.48 \pm 0.08$ & $0.39 \pm 0.07$ & $0.71 \pm 0.21$ & $0.68 \pm 0.13$ & $0.68 \pm 0.17$ \\
\hline & 3.44 & 2.42 & 3.18 & 4.15 & 4.17 & 3.89 \\
\hline & $5.80 \pm 0.62$ & $6.01 \pm 0.79$ & $6.27 \pm 0.68$ & $1.62 \pm 0.19^{c)}$ & $1.81 \pm 0.21^{c)}$ & $1.77 \pm 0.22^{c)}$ \\
\hline & \multicolumn{3}{|c|}{$\mathrm{PD}$} & & & \\
\hline & Cerebral cortex & Hippocampus & Thalamus & \\
\hline$A U C_{0-2}(\mu \mathrm{g} \cdot \mathrm{h} / \mathrm{g})$ & $9.15 \pm 1.40$ & $8.98 \pm 1.92$ & $9.28 \pm 1.65$ & & & \\
\hline$M R T(\mathrm{~h})$ & $1.00 \pm 0.08$ & $0.99 \pm 0.13$ & $1.03 \pm 0.05$ & & & \\
\hline$A U C_{\text {brain }} / A U C_{\text {plasma }}(\%)^{b)}$ & 54.03 & 53.03 & 54.80 & & & \\
\hline$k_{\mathrm{e}}\left(\mathrm{h}^{-1}\right)$ & $0.235 \pm 0.025^{()}$ & $0.273 \pm 0.028^{c)}$ & $0.162 \pm 0.019^{()}$ & & & \\
\hline
\end{tabular}

Each value represents the mean \pm S.D. of 3 rats. a) Dose: $\left.30 \mathrm{mg} / \mathrm{kg} . \quad b)\left(A U C_{\text {brain } 0-2 \mathrm{~h}} / A U C_{\text {plasma } 0-2 \mathrm{~h}}\right) \times 100 . \quad c\right) p<0.05$ versus the values for AP.

Table 2. Brain to Plasma Concentration Ratio ${ }^{a)}$ for AP and PD after I.v. and Oral Administration

\begin{tabular}{cccccc}
\hline \hline & & \multicolumn{4}{c}{ Time (h) after dosing } \\
\cline { 3 - 6 } & Drug & 0.5 & 1 & 2 & 4 \\
\hline I.v. & AP & 0.094 & $-{ }^{b)}$ & $-^{b)}$ \\
& PD & 0.591 & 0.640 & 0.560 & \\
Oral & AP & 0.300 & $-{ }^{b)}$ & $--^{b)}$ & $-^{b)}$ \\
& PD & 0.431 & 0.181 & 0.135 & 0.101 \\
\hline
\end{tabular}

a) Mean cerebral cortex level $(\mu \mathrm{g} / \mathrm{g}) /$ mean plasma level $(\mu \mathrm{g} / \mathrm{ml})$. b) Not calculated for low AP level in plasma, values below the detection limit.

transport system. Since PD has imino and keto groups in the molecule and is structurally similar to proline, there is the possibility that this compound could be transported across the BBB by a neutral amino acid carrier system. On the other hand, the reason why ABA was undetected in the brain is probably due to the high polarity of the metabolite, because the compound has a secondary amino group and a carboxylic acid, leading to rapid elimination of the metabolite from the circulation system after i.v. and oral administrations. ${ }^{1)}$

The brain (cerebral cortex)/plasma concentration ratios for AP and PD are shown in Table 2. The ratio for PD was also much larger than that for AP. This indicates the facile transport of PD across the BBB to the brain.

Oral Administration The concentration profiles of AP, $\mathrm{PD}$ and $\mathrm{AA}$ in 3 brain regions following oral administration $(50 \mathrm{mg} / \mathrm{kg})$ of the parent drug are depicted in Fig. 2. The pharmacokinetic parameters are shown in Table 3. Appreciable concentrations of AA and PD were observed in the brain regions, but were followed by rapid elimination. AP was also detected at low levels in the tissues. The concentration-time profiles of AP and AA in the brain regions after oral administration were similar to those after i.v. dosing. The $A U C_{\text {brain }}$ $A U C_{\text {plasma }}$ ratios of these compounds after oral dosing were $48-59 \%$ for AP, $21-22 \%$ for PD and $10-11 \%$ for AA. However, the ratio for AP may be a rough value because the $A U C_{\text {plasma }}$ was calculated from only 2 low plasma levels after oral administration. ${ }^{1)}$ The $F$ values of AP however were extremely small (below $0.2 \%$ ). The $A U C_{\text {brain }} / A U C_{\text {plasma }}$ ratio of $\mathrm{PD}$ was also more than that for AA. This data agreed well with the report that the highest level of PD among 4 metabolites was observed in rat brain $30 \mathrm{~min}$ after oral dosing of ${ }^{14} \mathrm{C}$-AP at a $50 \mathrm{mg} / \mathrm{kg}$ dose. ${ }^{4)}$ The elimination pattern of $\mathrm{PD}$ in the brain after oral administration was different from that after i.v. dosing. This difference would be due to the difference in the drug concentrations in plasma after both administrations (high and sustained levels after i.v. dosing, and low levels and comparatively rapid elimination after oral dosing), as shown previously. ${ }^{1)}$

AP and PD are pharmacologically active, but AA is not. ${ }^{2)}$ The concentrations of PD in the brain after both i.v. and oral administrations were much higher than those of AP. Therefore, this suggests that the clinical efficacy of dosed AP might partly be explained by PD penetrating into the brain. Additionally, it would be reasonable to assume that PD might be hardly converted from AP in the brain and PD itself would be transported across the BBB to the brain, since extremely low levels $(0.1 \mu \mathrm{g} / \mathrm{g})$ of AP in the brain 30 min after oral dosing $^{4)}$ and relatively low plasma concentrations, which were below the detection limit $1 \mathrm{~h}$ after i.v. dosing, were observed in rats. ${ }^{1)}$

In Situ Brain Perfusion The high and sustained levels of PD in the brain obtained in this study led us to test the possibility of carrier transport through the BBB. Several investigators have demonstrated the presence of carrier-mediated mechanisms responsible for the ready influx of amino acids, peptides, and some drugs into the brain. ${ }^{9)}$ Since PD has imino and keto groups in the molecule, and is somewhat similar to proline, there is the possibility that PD is transported across the BBB to the brain by a neutral amino acid carrier system (A system ${ }^{10)}$ ). We investigated the transport of PD using the in situ rat brain perfusion technique. ${ }^{6)}$ Table 4 shows the effects of various amino acids and an inhibitor of active transport on PD transport. PD transport across the BBB was not inhibited by coperfusion with L-proline and Lalanine, which are transported by the A system. Large amino acids, L-phenylalanine, L-leucine ${ }^{11)}$ and L-DOPA, an inhibitor of the large neutral amino acid carrier system (L system), ${ }^{12)}$ also did not affect the transport of PD. The sensitivity of the carrier to competition among neutral amino acid and PD for uptake is an important factor in determing the efficiency with which PD can be delivered to the brain. However, these data 
Cerebral cortex

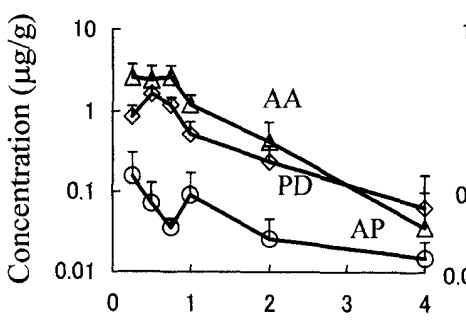

Hippocampus

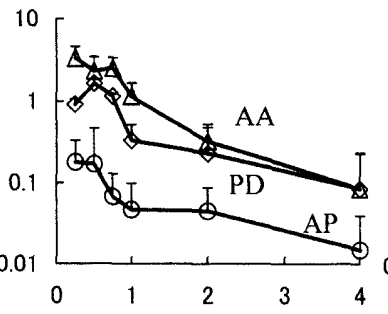

Time (h)
Thalamus

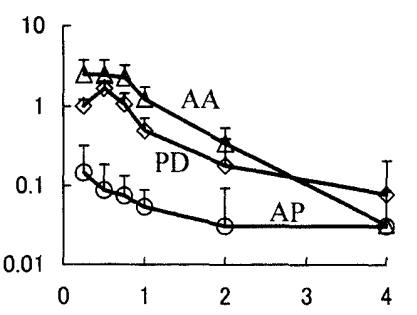

Fig. 2. Brain Concentration-Time Curves for AP and Its Metabolites after Oral Administration of AP

The dose of AP was $50 \mathrm{mg} / \mathrm{kg}$. Points and vertical bars represent the mean \pm S.D. of 5 rats.

Table 3. Pharmacokinetic Parameters of AP and Metabolites in Brain after Oral Administration of AP

\begin{tabular}{|c|c|c|c|c|c|c|}
\hline & \multicolumn{3}{|c|}{ AP } & \multicolumn{3}{|c|}{$\mathrm{AA}$} \\
\hline & Cerebral cortex & Hippocampus & Thalamus & Cerebral cortex & Hippocampus & Thalamus \\
\hline \multirow{6}{*}{$\begin{array}{l}A U C_{0-4}(\mu \mathrm{g} \cdot \mathrm{h} / \mathrm{g}) \\
M R T(\mathrm{~h}) \\
A U C_{\text {brain }} / A U C_{\text {plasma }}(\%)^{b)} \\
F(\%)\end{array}$} & $0.177 \pm 0.021$ & $0.215 \pm 0.024$ & $0.188 \pm 0.271$ & $3.371 \pm 0.382$ & $3.357 \pm 0.354$ & $3.157 \pm 0.361$ \\
\hline & $1.25 \pm 0.25$ & $1.24 \pm 0.23$ & $1.32 \pm 0.31$ & $0.93 \pm 0.17$ & $0.90 \pm 0.15$ & $0.90 \pm 0.15$ \\
\hline & $(48.3)^{c)}$ & $(58.7)^{c)}$ & $(51.4)^{c)}$ & 10.94 & 10.90 & 10.25 \\
\hline & 0.10 & 0.12 & 0.10 & - & - & - \\
\hline & \multicolumn{3}{|c|}{$\mathrm{PD}$} & & & \\
\hline & Cerebral cortex & Hippocampus & Thalamus & & & \\
\hline$A U C_{0-4}(\mu \mathrm{g} \cdot \mathrm{h} / \mathrm{g})$ & $1.674 \pm 0.323$ & $1.567 \pm 0.486$ & $1.594 \pm 0.409$ & & & \\
\hline$M R T(\mathrm{~h})$ & $1.02 \pm 0.27$ & $1.00 \pm 0.42$ & $0.96 \pm 0.33$ & & & \\
\hline$A U C_{\text {brain }} / A U C_{\text {plasma }}(\%)^{b)}$ & 22.20 & 21.14 & 20.78 & & & \\
\hline
\end{tabular}

Each value represents the mean \pm S.D. of 5 rats. a) Dose: $50 \mathrm{mg} / \mathrm{kg}$. b) $\left.\left(A U C_{\text {brain } 0 \cdots 4 \mathrm{~h}} / A U C_{\text {plasma } 0-4 \mathrm{~h}}\right) \times 100 . \quad c\right)$ The values may be approximate because of the low plasma levels after oral administration.

Table 4. The Effect of Amino Acid Carrier Substrates on Permeability of PD to the Cerebral Cortex

\begin{tabular}{cc}
\hline & Concentration in cerebral cortex $(\mu \mathrm{g} / \mathrm{g})$ \\
\cline { 2 - 2 } Amino acid and inhibitor ${ }^{a)}$ & \multicolumn{1}{c}{ Immediately after infusion } \\
\hline None (control) & $42.51 \pm 5.01$ \\
L-Proline & $49.22 \pm 10.36$ \\
L-Alanine & $41.99 \pm 7.30$ \\
L-Leucine & $44.29 \pm 12.21$ \\
L-Phenylalanine & $48.74 \pm 11.23$ \\
L-DOPA & $40.71 \pm 6.98$ \\
\hline
\end{tabular}

All values are expressed as the mean \pm S.D. of 4 rats. a) The concentration of amino acid in the perfusate was $0.1 \mathrm{M}$ except for L-DOPA.

demonstrate that $\mathrm{PD}$ is not transported across the BBB by $\mathrm{A}$ and $\mathrm{L}$ systems with characteristics of an amino acid carrier. However, it is possible that another transporter of PD across the $\mathrm{BBB}$ exists. Further experiments will be required to demonstrate a possible transporter of PD.

In this experiment, the tissue concentrations, but not the clearance, of PD were shown to simply compare the brain concentration after infusion with that after i.v. or oral administration. In addition, the reason why a high concentration $(0.1 \mathrm{M})$ of amino acid carrier substrates was used was to prove unambigously the inhibitory effect of substrate.
In conclusion, the concentrations of AP and its metabolite, $\mathrm{AA}$, in 3 brain regions rapidly declined after i.v. administration of AP, whereas PD levels were higher and more sustained than those of $\mathrm{AP}$ and $\mathrm{AA}$. The $A U C_{\text {brain }} / A U C_{\text {plasma }}$ ratio of PD thus was $53-55 \%$, in contrast to the low ratio of AP $(2.4-3.2 \%)$ and AA $(3.9-4.2 \%)$. On oral administration of $\mathrm{AP}$, the $A U C_{\text {brain }} / A U C_{\text {plasma }}$ ratio of $\mathrm{PD}$ was also higher than that of AA. Brain uptake of AP and AA was influenced by passive diffusion. We could not demonstrate that $\mathrm{PD}$ was transported across the BBB by a neutral amino acid carrier system.

\section{REFERENCES}

1) Ogiso T., Iwaki M., Tanino T., Ikeda K., Paku T., Horibe Y., Suzuki H., J. Pharm. Sci., 87, 594-598 (1998).

2) Kubota A., Furuya I., Kurasawa M., Yakuri To Chiryo, 14, 721-726 (1986).

3) Kubota A., Kurasawa M., Furuya I., Yakuri To Chiryo, 14, 713-720 (1986).

4) Nakayama S., Ichihara S., Ichihara Y., Sakata H., Tomisawa H., Tateishi M., Yakuri To Chiryo, 14, 795 -811 (1986).

5) Ichihara S., Sakata H., Nakayama S., Ichihara Y., Tomisawa H., Tateishi M, Yakuri To Chiryo, 14, 813-824 (1986).

6) Takasato Y., Rapoport S. I., Smith Q. R., Am. J. Physiol., 247, H484493 (1984). 
7) Gibaldi M., Perrier D. (eds.), "Pharmacokinetics," 2nd ed., Marcel Dekker, New York and Bassel, 1982, pp. 84-111 and 409-417.

8) Budavari S. (ed.), The Merck Index, 12th ed., Merck \& Co., New Jersey, 1996, p 112.

9) Tsuji A., "Trends and Future Perspectives in Peptide and Protein Drug Delivery," ed. by Lee V. H. L., Hashida M., Mizushima Y., Harwood
Academic Publishers, Chur, Switzerland, 1995, pp. 153-174.

10) Betz A. L., Goldstein G. W., Science, 202, 225-227 (1978).

11) Pardridge W. M. "Directed Drug Delivery;" ed. by Borchardt R. T., Repta A. J., Stella V. J., Humana Press, Clifton, New Jersey, 1985, pp. $83-96$.

12) Audus K. L., Borchardt R. T., J. Neurochem., 47, 484-488 (1986). 